\title{
It takes two to tango
}



\author{
Eco-Evolutionary \\ Dynamics \\ by Andrew P. Hendry \\ PRINCETON \\ UNIVERSITY PRESS: \\ 2016. 416PP. US\$65
}

E cology has traditionally been portrayed as a ballroom orchestra playing the rhythm that evolution dances to. Recent evidence, however, suggests that sometimes the dancer calls the tune, and may even dare to tango with the conductor. Thus, as in tango dancing where partners move in synch, sometimes parallel, sometimes in opposition, ecology and evolution are intertwined. The term eco-evolutionary dynamics describes the interplay between ecological and evolutionary processes on a short or contemporary timescale. Eco-Evolutionary Dynamics by Andrew P. Hendry presents an extensive review of this emerging field.

Feedbacks between ecological and evolutionary dynamics may appear obvious to some because this is the 'way the world works'. On a long timescale, no scientist doubts those feedbacks, which have been recognized for decades or even centuries. Thus, researchers who do not like to see ecology and evolution portrayed as independent boxes connected by many arrows may not like Hendry's perspective. However, the realization that evolution can affect ecological processes and vice versa on a short timescale has prompted a new series of experimental, observational and modelling studies to measure the strength of these feedbacks relative to other well-known drivers of ecological change. Whereas several of these insights come from lab studies where feedbacks from evolution to ecology might be amplified by a constant environment, Hendry focuses on natural environments and provides an excellent summary of the current state of knowledge on eco-evolutionary dynamics in the wild.

The book starts by presenting the rationale for expecting eco-evolutionary interactions on contemporary timescales. Then, it is divided into three main themes: six chapters cover the links from ecology to evolution, then three chapters are devoted to the effects of contemporary evolution on ecological dynamics, and finally two chapters review the 'underpinnings' of phenotypic traits and focus on two important drivers of phenotypic variation, namely genetic variation and plasticity. In addition to reviewing these topics, Hendry also identifies current areas of ambiguity and weakness in both theory and data, and often shares his own emergent insights. For example, he identifies several conditions for feedbacks between ecology and evolution to occur on a short timescale and makes predictions about which types of trait are more likely to be involved in those feedbacks.

Hendry's view of eco-evolutionary dynamics developed from his research on natural selection in wild species. His work on sockeye salmon was among the first to document 'rapid' evolution in nature. Hendry and his colleagues found that adjacent salmon populations of common ancestry developed reproductive isolation in just 13 generations after colonizing divergent reproductive environments. $\mathrm{He}$ has also documented evolutionary processes including adaptive divergence, gene flow and selection in Darwin's finches, guppies and sticklebacks. His book is the culmination of more than 20 years of research on the ecological drivers of evolutionary processes in nature.

Eco-evolutionary studies move on from such research by assessing the effects of evolutionary change on ecological dynamics at different levels of biological organization, including population dynamics, as well as community and ecosystem processes. This field has made ecologists and evolutionary biologists reconsider the assumption that evolution is slow and can be safely ignored by ecological models, or the assumption common in evolutionary models - that environmental variables are fixed. Although interest in measuring potential ecoevolutionary effects is mounting, empirical quantifications outside the lab are still scarce. Thus, much of the current literature on ecoevolutionary dynamics consists of conceptual frameworks, reviews of the few empirical examples and qualitative descriptions of when to expect that evolution should have quantifiable effects on ecology. The scope of this book on the possible feedbacks between evolution and ecology is therefore limited by the scarcity of empirical data from wild species. Most examples cited in the book are already well-known examples in evolutionary ecology including the response to drought of beak size in Darwin's finches, the changes in colour and life history traits in guppies due to predation or the effects of evolutionary diversification in threespine stickleback on ecosystem processes. The book, however, makes a very strong case for the value of conducting such studies in natural environments and points to key areas where progress is likely.

It is easy to find examples where evolution or ecology can have quantifiable effects on each other. To advance our understanding, however, we need to go beyond cherrypicking and test general predictions about the presence and importance (or the lack) of eco-evolutionary feedbacks. For example, to predict the dynamics of a harvested population, can I simply monitor the number of individuals, their sex, age and reproductive rate? Or should I also consider concurrent changes in morphology (let's say size) and life history (age at first reproduction) in response to size-selective harvest? Recent modelling studies in aquatic ecosystems suggest that we need to consider both, but empirical data to validate those models are rare, and it remains unknown whether or not those effects can be generalized to other ecosystems. It is critical that we move forward quickly to establish when evolutionary feedbacks are important for management and conservation of populations and ecosystems. Hendry's insights on the topic will hopefully stimulate new research on these pressing applied questions.

As recognized by the author in his preface, by trying to bring disparate fields together, one is forced into a trade-off between presenting a review that is "too technical for nonspecialists but not detailed enough for specialists". After all, entire books have been devoted to several of the topics presented here as chapters. Nevertheless, specialists in each of those fields might not be aware of progress made in other areas, and Hendry provides an excellent overview. The book will be a great read for students starting graduate courses in ecology or evolution. It will play the first notes that may inspire them to study the tango of nature, and hopefully take that study outside the ballroom. Let's dance.

\section{REVIEWED BY FANIE PELLETIER}

Fanie Pelletier is a professor at the Université de Sherbrooke, Québec, Canada.

e-mail:Fanie.Pelletier@USherbrooke.ca 